\title{
Meta-analysis of molecular response of kidney to ischemia reperfusion injury for the identification of new candidate genes
}

Dmitry N Grigoryev ${ }^{1,2,3^{*}}$, Dilyara I Cheranova ${ }^{1}$, Daniel P Heruth', Peixin Huang ${ }^{1}$, Li Q Zhang ${ }^{1}$, Hamid Rabb ${ }^{4}$ and Shui $\mathrm{Q} Y \mathrm{e}^{1,2,3}$

\begin{abstract}
Background: Accumulated to-date microarray data on ischemia reperfusion injury (IRI) of kidney represent a powerful source for identifying new targets and mechanisms of kidney IRI. In this study, we conducted a meta-analysis of gene expression profiles of kidney IRI in human, pig, rat, and mouse models, using a new scoring method to correct for the bias of overrepresented species. The gene expression profiles were obtained from the public repositories for 24 different models. After filtering against inclusion criteria 21 experimental settings were selected for meta-analysis and were represented by 11 rat models, 6 mouse models, and 2 models each for pig and human, with a total of 150 samples. Meta-analysis was conducted using expression-based genome-wide association study (eGWAS). The eGWAS results were corrected for a rodent species bias using a new weighted scoring algorithm, which favors genes with unidirectional change in expression in all tested species.

Results: Our meta-analysis corrected for a species bias, identified 46 upregulated and 1 downregulated genes, of which 26 (55\%) were known to be associated with kidney IRI or kidney transplantation, including LCN2, CCL2, CXCL1, HMOX1, ICAM1, ANXA1, and TIMP1, which justified our approach. Pathway analysis of our candidates identified "Acute renal failure panel" as the most implicated pathway, which further validates our new method. Among new IRI candidates were 10 novel ( $<5$ published reports related to kidney IRI) and 11 new candidates ( 0 reports related to kidney IRI) including the most prominent candidates ANXA2, CLDN4, and TYROBP. The cross-species expression pattern of these genes allowed us to generate three workable hypotheses of kidney IRI, one of which was confirmed by an additional study.
\end{abstract}

Conclusions: Our first in the field kidney IRI meta-analysis of 150 microarray samples, corrected for a species bias, identified 10 novel and 11 new candidate genes. Moreover, our new meta-analysis correction method improved gene candidate selection by identifying genes that are model and species independent, as a result, function of these genes can be directly extrapolated to the disease state in human and facilitate translation of potential diagnostic or therapeutic properties of these candidates to the bedside.

Keywords: Kidney, Ischemia reperfusion injury, Bioinformatics, Meta-analysis

\footnotetext{
* Correspondence: dgrigoryev@cmh.edu

'Division of Experimental and Translational Genetics, Department of

Pediatrics, Children's Mercy Hospitals and Clinics, University of Missouri

School of Medicine, Kansas City, MO, USA

${ }^{2}$ Department of Biomedical and Health Informatics, University of Missouri

School of Medicine, Kansas City, MO, USA

Full list of author information is available at the end of the article
} 


\section{Background}

There is accumulating evidence for the direct correlation between duration of donor organ ischemia and rejection rates $[1,2]$. Therefore, the development of protective therapies for ischemia reperfusion injury (IRI) of transplanted organs is of great importance. However, the mechanisms by which IRI predisposes to allograft injury are poorly understood. To capture this complex process, global gene expression profiling has been intensively utilized and its results made available to public. The accumulated data represent a remarkable substrate for the analysis of global molecular changes invoked by kidney IRI in different experimental settings. However, most of the kidney IRI data is generated using rodent experimental models. Therefore, we hypothesized that correction of meta-analysis of IRI-implicated genes for a species bias will not only improve new gene candidate selection, but will identify genes that are model and species independent. Therefore, the new diagnostic or therapeutic properties discovered for these genes will be easier translated to a clinical use.

\section{Results and discussion}

The 21 experimental settings used for our meta-analysis comprised 150 samples (65 shams and 85 IRIs). The combined data set had 12 post-ischemia time-points represented by five different platforms (ABI, Affymetrix, Agilent, Amersham, and Illumina) and four species (human, pig, rat, and mouse) and was stratified by the recovery time after ischemia (Table 1 ).

The cross-referencing microarray platforms resulted in universal 17,575 genes, which were analyzed by eGWAS. The eGWAS ranked genes by the likelihood that repeated differential expression for a given gene was due to chance, then controlled for multiple-hypothesis testing using Bonferroni threshold $\left(\mathrm{P}<2.84 \times 10^{-6}\right)$. The eGWAS identified 75 candidate genes (Figure 1, Additional file 1). To correct this result for species and platform bias, these 75 genes were cross-referenced against 116 candidates identified by the weighted scoring ( $W$-score) algorithm (Figure 2, Additional file 2). The resulting 47 genes were divided into three tiered candidate lists (Figure 2, Additional file 3). First tier contains 20 genes, which responded to IRI in all time points, or in 4 consequent time points (Table 1 , Figure 2). Second tier contains 23 genes, which responded to IRI in 3 consequent time points (1-36 h, $4 \mathrm{~h}-10$ days, or 24 h-3 month; Figure 2) and third tier contains 4 candidates, which responded to IRI only at 2 time points.

To evaluate, whether our tight filtering affected biological relevance of retained 47 gene candidates, we conducted Ingenuity Pathway Analysis (IPA). The IPA of 47 candidates identified Acute Renal Failure Panel as the top IRI affected pathway $(\mathrm{P}<0.000001$, Additional file 4). The pathway analysis for each time-point was conducted using significant candidates, identified by $W$-scoring: 430 , 684, 1069, 369, and 93 genes for 1-2 hours, 4-8 hours, 24-36 hours, 3-10 days; 3-5 month after IRI, respectively. The five sets of pathways were generated. The pathways, which were among the top three pathways in at least two consecutive time points are reported in Figure 3 by their significance: "Acute Renal Failure Panel", "Liver Necrosis/ Cell Death, Renal Necrosis/Cell Death", "Persistent Renal IRI", and "Hepatic Fibrosis". This result further validated our findings and addressed concern that our algorithm puts a strong filter on candidate gene selection, which might inappropriately restrict the biological relevance of the results.

To identify relevance of our candidates to kidney IRI we linked 47 genes to 3 terms: "kidney IRI", "kidney transplantation", and "kidney injury" using PubMatrix tool [3]. This approach identified 26 genes (55.3\%) as well established kidney IRI genes ( $\geq 5$ combined citations (CC) of 3 search terms), 10 (21.2\%) novel genes $(5>C C>0)$ with top candidates ADAMTS1, ANXA2, and TYROBP; and 11 (23.5\%) new genes $(C C=0)$ with top candidates $A R P C 1 B$, $E G R 2$, and CLDN4 (Additional file 3). The detailed analysis of 20 tier-one candidates revealed significant association of $70 \%$ (14 out of 20 genes) with kidney injury, IRI, or transplantation (Table 1), which once again confirmed biological feasibility of our method.

The comparison of 47 gene candidates that were selected by both eGWAS and $W$-scoring algorithms with 28 candidates selected by eGWAS but not $W$-scoring algorithms (Additional file 5) demonstrated that our algorithms filtered out genes with higher bias of rodent models and lower contribution of human models (39.3\% of filtered out by our algorithm genes were selected by eGWAS solely based on the rodent models and only $35.7 \%$ were selected using human data). On the other hand, among the W-scoring retained candidates only $14.9 \%$ of genes was selected based on rodent models and more than half of the retained candidates $(51.5 \%)$ were selected using human data (Additional file 5).

These findings suggest that expression patterns of gene candidates selected by the $W$-scoring algorithm are shared at higher degree amongst species, therefore they might have higher rates of success during further validation in different models. Three of these candidates allowed us to generate three workable hypotheses, one of which was confirmed by an additional study.

Genes in which the whole family was involved in kidney IRI became our primary candidates. The first such gene was $A N X A 2$ from the annexin A family. Its counterpart $A N X A 1$ is a well known kidney IRI gene $[4,5]$, which conformingly had the highest kidney IRI association P-value (Figure 1). Based on the knowledge that ANXA1 protects kidney against IRI via its potent neutrophil anti-migratory activity [4], one can predict the same 
Table 1 First tier IRI gene candidates

\begin{tabular}{|c|c|c|c|c|c|c|}
\hline \multirow[t]{2}{*}{ Gene } & \multirow[t]{2}{*}{ Symbol } & \multirow[t]{2}{*}{$x^{2} \mathrm{P}$ value } & \multirow[t]{2}{*}{ W score } & \multicolumn{3}{|c|}{ PubMatrix search terms } \\
\hline & & & & $\begin{array}{l}\text { Kidney } \\
\text { injury }\end{array}$ & $\begin{array}{l}\text { Kidney ischemia } \\
\text { reperfusion injury }\end{array}$ & $\begin{array}{c}\text { Kidney } \\
\text { transplantation } \\
\end{array}$ \\
\hline Lipocalin 2 & LCN2 & $2.36 \mathrm{E}-11$ & 33.3 & 314 & 38 & 57 \\
\hline TYRO tyrosine kinase binding protein & TYROBP & $5.54 \mathrm{E}-08$ & 22.3 & 2 & 1 & 1 \\
\hline Annexin A1 & ANXA1 & 4.52E-14 & 27 & 3 & 3 & 1 \\
\hline Chemokine (C-C motif) ligand 2 & CCL2 & $3.97 \mathrm{E}-11$ & 36 & 313 & 56 & 96 \\
\hline Heme oxygenase (decycling) 1 & HMOX1 & 3.97E-11 & 27 & 291 & 100 & 110 \\
\hline Clusterin & CLU & 3.97E-11 & 25.5 & 27 & 1 & 11 \\
\hline Early growth response 2 & EGR2 & $1.43 \mathrm{E}-10$ & 19 & 0 & 0 & 0 \\
\hline Cyclin-dependent kinase inhibitor $1 \mathrm{~A}$ & CDKN1A & $2.34 \mathrm{E}-10$ & 26 & 83 & 16 & 28 \\
\hline Activating transcription factor 3 & ATF3 & $1.38 \mathrm{E}-09$ & 29 & 5 & 5 & 1 \\
\hline Chemokine (C-X-C motif) ligand 1 & CXCL1 & $1.38 \mathrm{E}-09$ & 25 & 32 & 18 & 11 \\
\hline ADAM metallopeptidase with TS1 & ADAMTS1 & $1.75 \mathrm{E}-09$ & 29 & 2 & 1 & 1 \\
\hline RAS, dexamethasone-induced 1 & RASD1 & 5.33E-09 & 18 & 1 & 1 & 1 \\
\hline TIMP metallopeptidase inhibitor 1 & TIMP1 & 8.11E-09 & 31 & 162 & 57 & 163 \\
\hline TNF receptor superfamily, member $12 \mathrm{~A}$ & TNFRSF12A & 9.88E-09 & 32.5 & 9 & 1 & 2 \\
\hline Jun proto-oncogene & $J U N$ & $5.52 \mathrm{E}-08$ & 23 & 134 & 37 & 36 \\
\hline Filamin A, alpha & FLNA & $5.52 \mathrm{E}-08$ & 19 & 0 & 0 & 0 \\
\hline Intercellular adhesion molecule 1 & ICAM1 & $5.52 \mathrm{E}-08$ & 18 & 352 & 131 & 244 \\
\hline Secreted phosphoprotein 1 & SPP1 & $5.54 \mathrm{E}-08$ & 24 & 95 & 7 & 22 \\
\hline Lectin, galactoside-binding, soluble, 3 & LGALS3 & $5.54 \mathrm{E}-08$ & 21 & 11 & 4 & 3 \\
\hline Baculoviral IAP repeat-containing 3 & $\mathrm{BIRC3}$ & $1.66 \mathrm{E}-06$ & 14 & 1 & 0 & 1 \\
\hline
\end{tabular}

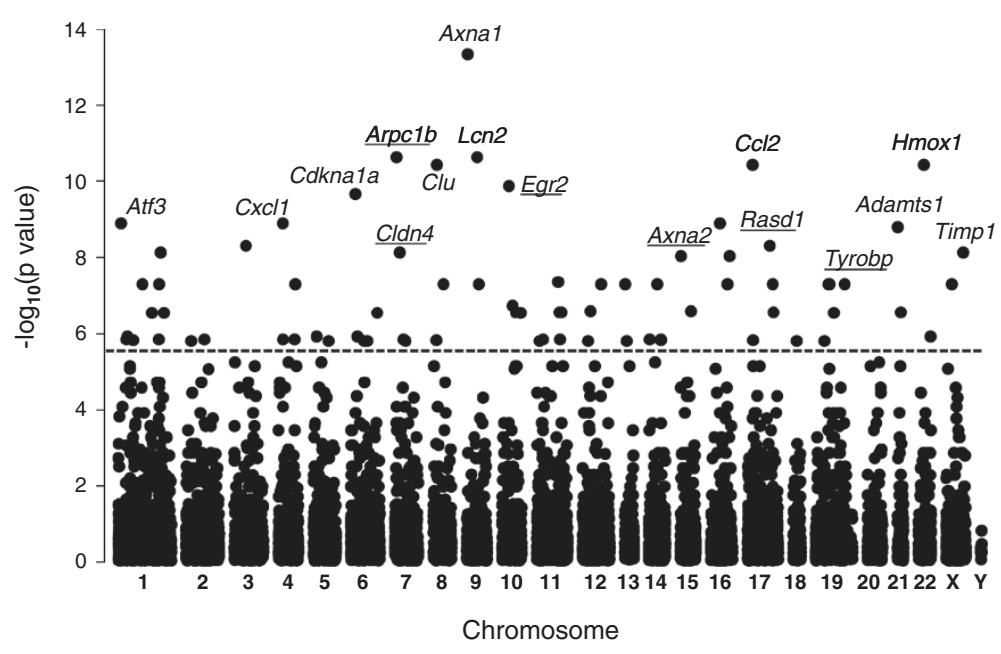

Figure 1 eGWAS for kidney IRI using a $x^{2}$ analysis. Plot of ( $-\log _{10} P$ value) ( $y$ axis) by chromosomal location ( $x$ axis). $P$ values for each gene were calculated for 21 microarray experiments with 65 sham-operated or pre-transplant control microarray samples and 85 IRI or post-transplant microarray samples as the likelihood of finding repeated differential expression compared with expected using $x^{2}$ analysis. Out of tested 17,575 genes 75 genes demonstrated a significant differential expression. The dotted line indicates the Bonferroni threshold $\left(P=2.84 \times 10^{-6}\right)$. The genes symbols indicate genes that are most significantly associated with kidney IRI, where underscored symbols indicate new candidate genes. 


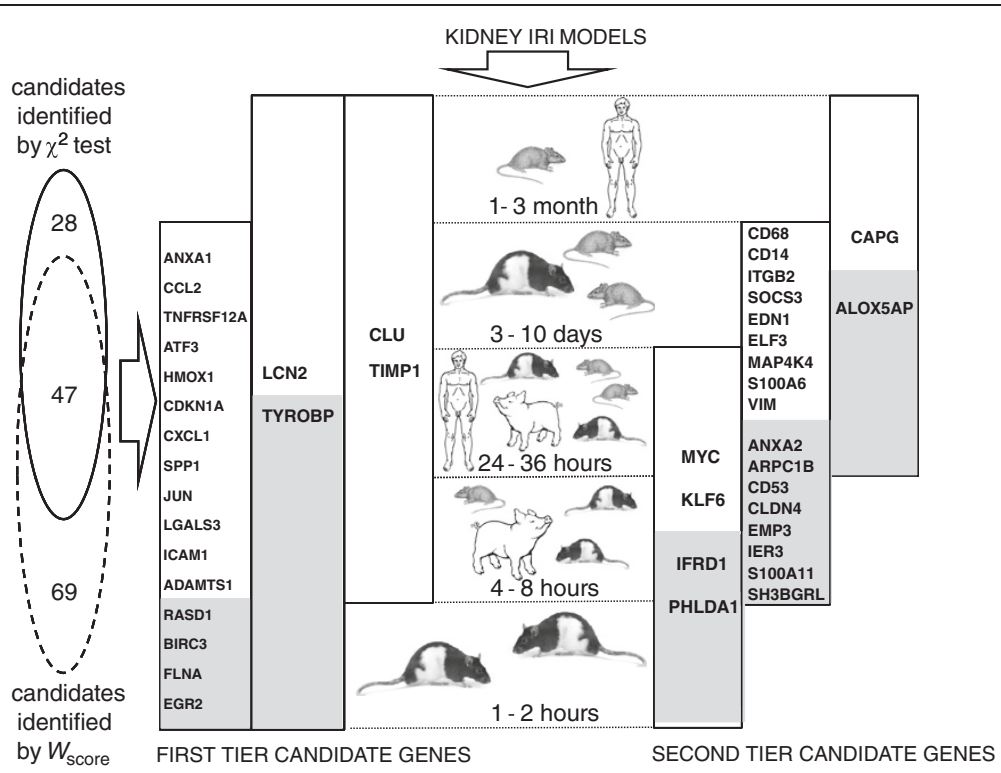

Figure 2 Analytical arrangement of available genomics studies of IRI in kidney and resulting lists of major IRI-associated candidate genes. Seventeen global transcriptional profiles were grouped into five clusters according to post-ischemia time. The species composition of each cluster is represented by a corresponding mammal. Vertical bars represent extend of significant expression of a particular gene throughout the five time-points. 46 upregulated and 1 downregulated candidate genes were selected by cross-referencing gene lists generated with $x^{2}$ test and $W$-scoring procedures (Venn diagram on the left). First tier candidate genes are depicted on the left and were significantly affected by IRI in at least four consecutive time-points. Each bar is filled with the corresponding gene symbol; where the open part of a bar is populated with the well known kidney IRI genes and grey area of the bar cover novel and new kidney IRI genes. The second tier candidate genes are listed on the right with the same color coding. The full names of depicted genes can be found in Table 1 (first tier) and Additional file $3 .{ }^{*}$ The 4 genes from the third tier candidates (JUNB, $\downarrow U P B 1, C X C L 10, C E B P D)$ are not included into the figure.

function for the new candidate $A N X A 2$. However, the other well studied annexin $A N X A 5$, exerts its antiinflammatory effect via a different mechanism: by blocking phosphatidylserine dependent inflammatory signaling [5]. Therefore, we hypothesize that $A N X A 2$ will alleviate kidney IRI via its antiinflamatory action, but by which mechanism remains to be investigated. Yet, the reported association of ANXA2 with tubular injury [6] will steer the future studies towards the epithelial component of kidney.

Our next candidate CLDN4 was from the claudin family. This family has the same basic function of maintaining epithelial barrier integrity and controlling paracellular

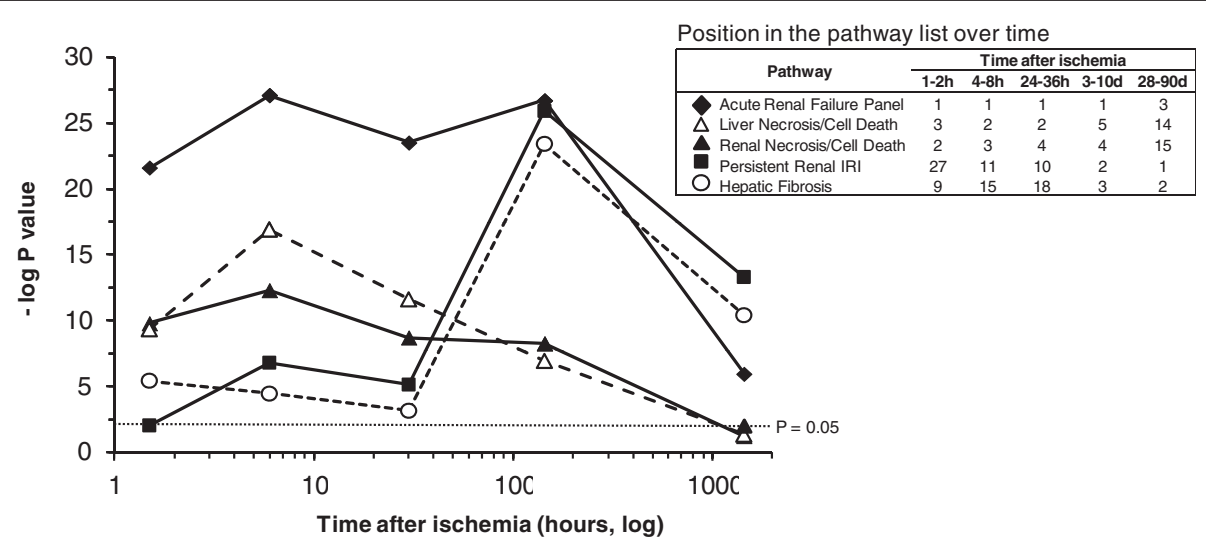

Figure 3 Ischemia time related pathway analysis of universal interspecies IRI genes. Genes that were significantly affected by IRI in more than one species at each selected time-point were analyzed by Ingenuity pathway tool. The top three significant pathways from each time-point were identified, and those that were present among top pathways at least twice were plotted against time-course of the IRI. The $y$ axis represents negative log of p-value assigned by Ingenuity algorithm (Fisher Exact test) to a pathway. The $p$-value that corresponds to numeric 0.05 is depicted by the dashed line. The $x$ axis represents time course in $\log _{10}$ format. The position of each depicted pathway in the list of pathways is shown in the insert table. 
transport via regulation of tight junctions between neighboring cells. Claudins are highly conserved throughout evolution and are located in close proximity in the human genome [7]. Our candidate CLDN4 is located within $50 \mathrm{~kb}$ of CLDN3 on chromosome 7. Furthermore, the CLDN3/ CLDN4 tandem demonstrates a coordinate expression in several normal and neoplastic tissues [8]. This was concordant with our data (Figure 4A). Although CLDN3 did not satisfy Bonferroni threshold, it was ranked by our W-scoring algorithm higher (rank 22) than CLDN4 (rank 78) (Additional file 2). Moreover, the overall trend in its expression pattern was close to that of CLDN4 (Figure 4A). The slight differences in expression of the tandem CLDN3/CLDN4 members might be attributed to the additional cortex location of $C L D N 3$ where expression pattern of CLDN3 might differ (Figure $4 B$ ).

Based on the report that the renal protective agent lypoxin, which is thought to exert its protective effects via significant downregulation of numerous IRI-inducible genes, including claudins [9], we hypothesized that the suppression of claudins, which delays the restoration of epithelial integrity, might be beneficial during IRI. We propose a mechanism where the leaky intercellular connections permits nonspecific paracellular dumping of excess inflammatory cytokines into the tubular urinary flow, thus clearing kidney tissue from injurious molecules and decreasing inflammation.

The last candidate, TYROBP, had the highest $W$-score among novel/new candidates and satisfied Bonferroni threshold (Table 1 and Figure 1). This gene was also the only new candidate expressed at all time points (Figure 2). $T Y R O B P$ is expressed in circulating immune cells and plays a crucial role in natural killer (NK) cell signaling. To date the known tissue specificity of TYROBP is restricted to the immune system and this gene was indirectly linked to kidney IRI via studies of non-specific response by circulating immune cells [10]. There are no reports on the expression of TYROBP in kidney tissues (http://www. genecards.org). However, our observations suggested otherwise. Analyzing IRI effects in cortex and medulla of rat kidney reported by Krishnamoorthy et al. [11] we noticed an unexpected pattern in expression of TYROBP. Knowing that $>90 \%$ of kidney blood flow occurs in the cortex; we anticipated that infiltration of immune cells will occur mainly in this compartment of the kidney, therefore, the expression of TYROBP will be higher in the cortex. Surprisingly, we observed the opposite (Figure 4C). Moreover, the marker of NK, CD160 [12] was not changed in either the cortex or in the medulla, suggesting that the TYROBP signal is coming not from immune cells, but rather from kidney tissues. This stipulation was indirectly supported by the fact that expression of TYROBP was detected in liver tissue as well [13], an important link, since the transcriptional response of liver to IRI in our study was similar to that of kidney (Figure 3).

To test this hypothesis we conducted real time PCR studies in the human kidney cell line HK-2. We simulated IRI in this cell using oxygen-glucose deprivation followed by recovery in regular media. This study demonstrated detectable expression of TYROBP in human kidney cells (Additional file 6: Figure S1) and its responsiveness to simulated IRI (Figure 5).

Thus, TYROBP is a fascinating therapeutic target, the inhibition of which might protect the kidney from NKs.

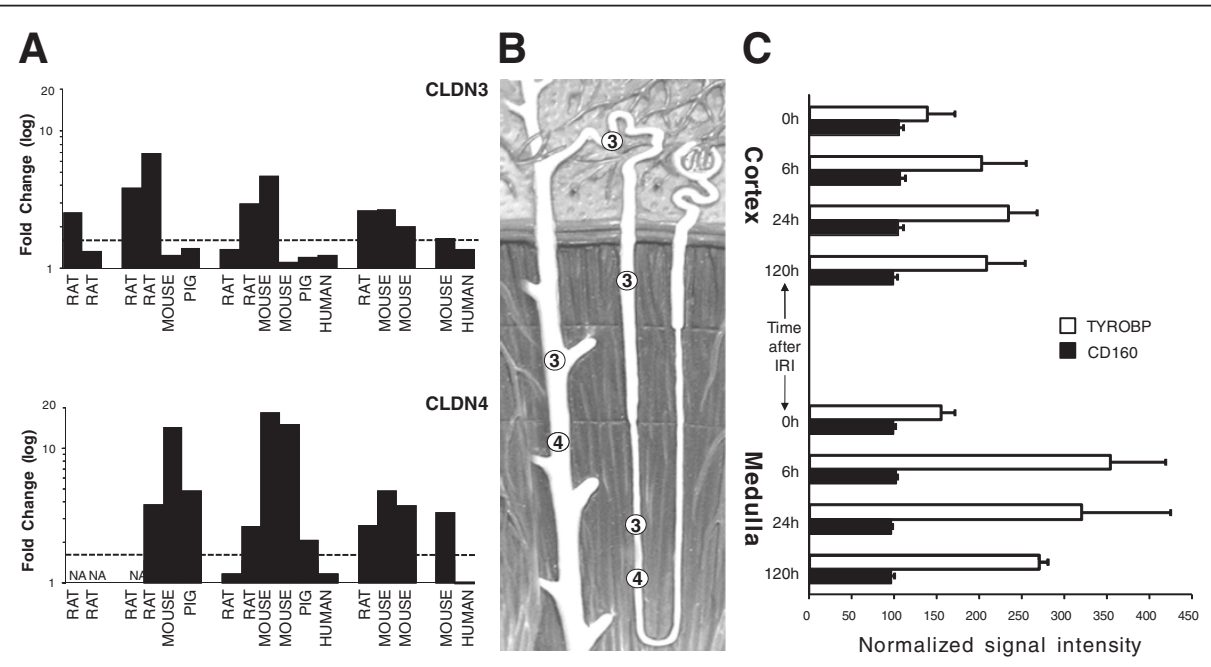

Figure 4 IRI-induced expression pattern of novel IRI candidate genes CLDNs and TYROBP. Panel A. The expression profiles ( $Y$ axis) of CLDN3 and CLDN4 are presented according to the tested time points ( $X$ axis). Fold changes (black bars) for each species (labeled on the bottom) are expressed in $\log _{10}$ values. The location of each claudin in the distal nephron is depicted in Panel $\boldsymbol{B}$ (each number corresponds to claudin nomenclature). Panel B. Diagram of nephron. Panel $\boldsymbol{C}$. The pixilated expression values of TYROBP and CD160 genes in cortex and medulla of kidney is represented by horizontal bars. The error bars are standard deviations among 3 samples. 


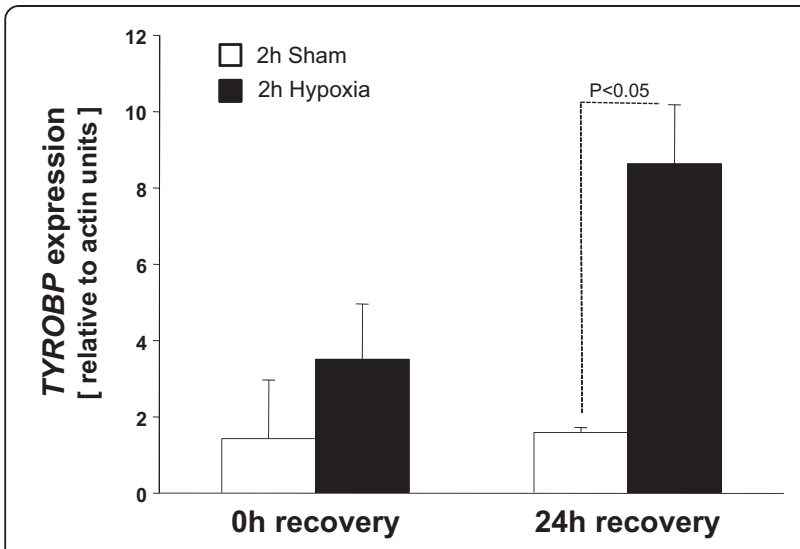

Figure 5 Expression of TYROBP genes in hypoxic human kidney (HK-2) cells identified by time PCR. The relative message abundance of TYROBP was detected by real time RT-PCR. The total RNA was obtained from HK-2 cells collected at 0 or 24 hours after hypoxia. The gene expression values are represented by relative to actin- $\beta$ expression units (mean (2 independent experiments) $\pm S D$ ). Changes in the gene expression between sham treated and hypoxia treated cells were evaluated by unpaired t-test and $\mathrm{P}<0.05$ was considered significant.

\section{Conclusions}

In the presented study, we investigated the response of four different biological systems to kidney ischemia using new pipeline eGWAS - W-scoring. This algorithm allowed us to identify reliable IRI candidate genes and select new promising candidates for further studies.

Based on our findings we were able to propose three new hypotheses and provide supporting evidence for one of them.

In summary, our first in the field kidney IRI metaanalysis of 150 microarray samples using eGWAS in combination with our new algorithm of correcting species contribution to the IRI molecular signal, identified 10 novel and 11 new gene candidates, of which 3 described above can lead to discovery of new unsuspected mechanisms of IRI. Moreover, a decreased bias of these genes for the most popular kidney IRI researched rodent model will facilitate investigation of their functions in non-rodent models and accelerate translation of their potential diagnostic and/or therapeutic properties to the bedside.

\section{Methods}

Data

The kidney IRI datasets were uploaded from the public genomics data repositories Gene Expression Omnibus (GEO, NCBI, (http://www.ncbi.nlm.nih.gov/geo) and ArrayExpress (EBI, http://www.ebi.ac.uk/arrayexpress). The term "kidney ischemia" was submitted to the database and 136 entries were returned (as of April 2012), 24 of which were data series and the remaining data were either array platforms or individual samples.

The inclusion criteria for retrieved data were: 1) the array samples must represent genome wide studies of the whole kidney by established microarray platforms with the number of interrogated sequences $>5,000$, thus excluding pathway oriented and small custom platforms, which will introduce tissue or biological process bias; 2) the experimental settings must have untreated (placebo or sham) and unmodified (wild type) subjects; 3 ) the IRI model must be based on the bilateral kidney injury (i.e. bilateral clamping), the unilateral models were excluded; and 4$) \mathrm{n} \geq 5$ per each comparison group (sham + IRI) group.

The studies that satisfied our inclusion criteria are listed in Table 2. Six data series with assigned GEO ID included 9 independent time points of IRI. The specific GEO sample IDs (GSM), which were used for this study

Table 2 Summary of microarray data used for meta-analysis

\begin{tabular}{|c|c|c|c|c|c|c|c|}
\hline \multirow{2}{*}{ Authors } & \multirow{2}{*}{ GEO series } & \multirow{2}{*}{ Species } & \multicolumn{5}{|c|}{ Effects of ischemia reperfusion injury } \\
\hline & & & Immediate & Early & Intermediate & Prolonged & Distant \\
\hline Kusaka et al. [14] & GSE5104 & Rat & $1 \mathrm{~h}$ & & & & \\
\hline Yuen et al. [15] & GSE3219 & Rat & $2 \mathrm{~h}$ & $8 \mathrm{~h}$ & & & \\
\hline Hanto et al. [16] & GSE14373 & Pig & & $4 \mathrm{~h}$ & $24 \mathrm{~h}$ & & \\
\hline Grigoryev et al. [19] & $N R^{*}$ & Mouse & & $6 \mathrm{~h}$ & $36 \mathrm{~h}$ & & \\
\hline \multirow[t]{2}{*}{ Krishnamoorthy et al. [11] } & GSE27274 & Rat Crtx & & $6 \mathrm{~h}$ & $24 \mathrm{~h}$ & $5 d$ & \\
\hline & & Rat Mdl & & $6 \mathrm{~h}$ & $24 \mathrm{~h}$ & $5 d$ & \\
\hline \multirow[t]{2}{*}{ Viñas et al. [17] } & GSE9943 & Rat BN & & & $24 \mathrm{~h}$ & & \\
\hline & & Rat SD & & & $24 \mathrm{~h}$ & & \\
\hline Grigoryev et al. [21,22] & NR & Human & & & $24 \mathrm{~h}$ & & \\
\hline Liu et al. [20] & NR & Mouse & & & $24 \mathrm{~h}$ & & \\
\hline Ko et al. [23] & Pending & Mouse & & & & $3 d, 10 d$ & $28 d$ \\
\hline Naesens et al. [18] & GSE11166 & Human & & & & & $90 d$ \\
\hline
\end{tabular}

* - not reported to GEO, Crtx - cortex, Mdl - medulla, BN - Brown Norway strain, SD - Sprague-Dawley strain. 
and a brief description of experimental settings is listed below starting with the author.

Kusaka et al. [14]: (Rat whole kidney isografts from brain dead donors, shams $\mathrm{n}=3$; ischemia 6 hours and reperfusion 1 hour $\mathrm{n}=3$ ) GSM115035, GSM115037, GSM115038.

Yuen et al. [15]: (Rat whole kidney, both renal pedicles were clamped for $40 \mathrm{~min}$, reperfused for $2(\mathrm{n}=4)$ or $8(\mathrm{n}=3)$ hours with 3 common shams) GSM71831, GSM71835, GSM72108, GSM72109, GSM72114, GSM72117, GSM72229, GSM72253, GSM72281, GSM72502.

Hanto et al. [16]: (Pig whole kidney, renal pedicles were clamped for 1 hour and reperfused for $4(n=4)$ or $24(\mathrm{n}=4)$ hours with 2 common controls) GSM359391, GSM359392, GSM359393, GSM359395, GSM359397, GSM359399, GSM359401, GSM359403, GSM359405, GSM359407.

Krishnamoorthy et al. [11] (Rat cortex and medulla from ischemic (bilateral 20 min clamping) kidneys, which were reperfused for 6 hours $(n=6) 24$ hours $(n=6)$ and 120 hours $(\mathrm{n}=6)$ with 6 common shams. The microarray data was generated for cortex and medula: GSM674258-81.

Viñas et al. [17]: (Rat whole kidney from SpragueDawley and Brown Norway strains, bilateral clamping for $45 \mathrm{~min}$, reperfusion for 24 hours $(\mathrm{n}=3)$ with 3 shams $)$ GSM251560-61, GSM251586, GSM251588, GSM251591, GSM251593-95, GSM280410-11, GSM280416-17.

Naesens [18]: (Human kidney biopsies before $(\mathrm{n}=$ 14) and 90 days after $(\mathrm{n}=14)$ transplantation $)$ GSM281084, GSM281259, GSM281276-77, GSM28134647, GSM281351-52, GSM281667-73.

The other 4 gene expression profiles were generated, studied and reported by our group [19-23]. Grigoryev et al: Mouse whole kidney, bilateral clamping for 1 hour, reperfusion for $6(n=3)$ or $36(n=3)$ hours with corresponding shams ( $\mathrm{n}=5, \mathrm{n}=3$, respectively) [19].

Lui et al: Mouse whole kidney, bilateral clamping for 30 min, reperfusion for 24 hours $(\mathrm{n}=3)$ with sham operated controls $(\mathrm{n}=3)$ [20].

Grigoryev et al: Human kidney biopsies, 11 paired samples before and 24 hours after transplantation $[21,22]$.

Ko et al: Mouse whole kidney, bilateral clamping for $30 \mathrm{~min}$, reperfusion for 3,10 , or 28 days $(\mathrm{n}=3$ for each time point) with corresponding sham operated controls (total $\mathrm{n}=9$ ) [23].

\section{Standardizing platforms}

All single channel arrays were reanalyzed using Significance Analysis of Microarrays (SAM 2.20) [24], which was conducted using 300 permutations without application of arbitrary restrictions [25], as described previously [26]. The fold change values from the multiple channel platform (GPL890) were used directly and $\log 2$ ratio p-values were used in place of $d$ score where $p<0.01$ was considered significant. The numeric q-values on other platforms were converted to $\log 2$ values to facilitate computing of average cross-platform q-value for a given gene. The log incompatible $\mathrm{q}=0$ we converted to the closest power fraction that SAM called above 0 . For example, the lowest fractional call for $\mathrm{q}$ in rat dataset from Vinas et al. was $\mathrm{q}=0.80$ and all other genes with higher significance had $\mathrm{q}=0$. We converted these 0 q-values to 0.1 . The lowest fractional call for q-value in rat dataset from Krishamoorthy et al. was $\mathrm{q}=0.02$ and all other genes with higher significance had a $\mathrm{q}$-value $=0$. We converted these 0 q-values to 0.01 .

The resulting data sets comprised 5 columns: combined probe IDs, gene name, gene symbol, d score, and fold change. The probe IDs across different microarray platforms for mouse, rat, and human were linked using array information library universal navigator (AILUN) tool (http://ailun.stanford.edu) [27], the probes that remained unmatched by AILUN and probes from pig arrays were linked to the AILUN created mouse-rat-human dataset via their gene symbol entries.

\section{Expression-based genome-wide association study (eGWAS)}

The eGWAS was conducted as described previously [28]. Briefly, to estimate differences between groups of samples from IRI subjects and sham controls, the d score, which denotes the standardized change in gene expression [28] was used (calculation of $\mathrm{d}$-score was performed using SAM 2.20 as described above). Genes with an absolute value of $d$ score $\geq 2$ or a fold change $\geq 2$ between shams and IRI were considered significantly dysregulated. There were total of 17,575 known genes across all microarray platforms. For every one of the 17,575 genes, the observed number of microarray experiments in which each gene was significantly dysregulated was derived. Then the $\mathrm{P}$ values from the number of positive/negative experiments for each gene and sum of the number of positive/negative experiments for all other genes was calculated using a $2 \times 2$ chi-square analysis or a Fisher's exact test as an alternative method, and ranked all of the genes according to their $\mathrm{P}$ value $\left(-\log _{10} \mathrm{P}\right)$.

\section{Weighted scoring algorithm}

Given that available data was skewed towards rodent models: 13 arrays for mouse and rat models and 4 arrays for pig and human models, there was a need for species bias correction. The straightforward approach of analyzing each species individually and then combining data will greatly decrease statistical power of meta-analysis. Therefore, we completed the meta-analysis of all arrays together and then cross-referenced obtained results with the unbiased gene list, which was generated using a 
weighted scoring algorithm modified from our previously reported approach [29]. Given that FC is heavily dependent on the platform (i.e. data submitted as RMA output provides relatively low FC values even for the most affected genes comparing to GCOS output of the same data) our algorithm favors the unidirectional gene expression over the magnitude of FC and uses the former as a multiplying factor and the later as a summation factor during score calculation. First we averaged fold change and $q$ values for the genes that had multiple probe sets on the same array, then the genes were scored based on the FC values in the $\log 2$ increments. The upregulated genes with $1.2 \leq \mathrm{FC}<2$ had score 1 ; $2 \leq \mathrm{FC}<4$ had score 2; $4 \leq \mathrm{FC}<8$ had score $3 ; 8 \leq$ $\mathrm{FC}<16$ had score 4; FC $>16$ had score 5 . The same method was used for the downregulated genes, where scores ranged from -1 to -5 .

The scores from different species were converted into the common weighted scores ( $W$-score) using our heuristic scoring algorithm [29]. The calculation was based on the score of individual expression signal $(m)$ and the number of tested species $(i)$ where this signal was detected. The $W$-score $(W)$ of a gene was then defined as a sum of $m$ reduced (penalized) by the unidirectional coefficient (bracketed expression of the formula). The unidirectional coefficient was computed as a total number of tested species reduced by the number of species, in which a given gene was not changed $\left(i_{n s}\right)$ and by the double number of species, in which a given gene was oppositely expressed; then divided by the number of tested species. Thus, if all species demonstrate unidirectional expression for a given gene, the $W$ of the gene is simply the sum of individual scores for each tested species.

$$
W=\sum_{\substack{i=1 \\ i \geq 2 i_{o p}}}^{m} m_{i}^{\mathrm{x}}\left(\frac{i-\left(i_{n s}+2 i_{o p}\right)}{i}\right)
$$

To eliminate the species bias all arrays from the same species were combined, scored using the described algorithm and converted back to the representative FC using the resulting score divided by the number of biological replicates. As an example we offer the description of scoring for the CCL2 gene at time point $24 \mathrm{~h}-36 \mathrm{~h}$ : $\mathrm{FC}_{\text {rat1 }}=2.0, \mathrm{FC}_{\text {rat2 }}=2.1$ gave $(2+2)^{*} 1 \rightarrow$ score $=4=>$ $4 / 2=2$ or $\mathrm{FC}=3.0]\left[\mathrm{FC}_{\text {mouse } 1}=1.3, \mathrm{FC}_{\text {mouse } 2}=7.8\right.$ gave $(1+3)^{*} 1 \rightarrow$ score $=4=>4 / 2=2$ or $\mathrm{FC}=3.0$. The scores of all four species were summed up and multiplied by the number of species: $\mathrm{FC}_{\text {human }}=2.8, \mathrm{FC}_{\mathrm{pig}}=21.4$, $\mathrm{FC}_{\text {rat }}=3.0, \mathrm{FC}_{\text {mouse }}=3.0[$ score $=(2+5+2+2) * 4]=44$.

The reducing (penalizing) system for the $W$ was based on the biological meaningfulness. For instance, the gene $A$ which was present on arrays for all four species but was upregulated in three of them and in the fourth species had no change in expression should be scored lower than gene $B$ which was upregulated in all four species, but scored higher than gene $C$, which was upregulated in three species but downregulated in the fourth species.

Assuming that all four species from the example above have $\mathrm{FC}=2$, then we will have the following three scores: $[2,2,2,2](2+2+2+2) \times 4 / 4 \rightarrow$ score $=8 ;[2,2,2,0](2+$ $2+2+0) \times(4-1) / 4 \rightarrow$ score $=4.5 ;[2,2,2,-2](2+2+$ $2-2) \times(4-2) / 4 \rightarrow$ score $=2$. The detailed example of scoring matrix is provided in Additional file 7.

For candidate gene selection throughout all time points the universal cutoff for the score value was set at 2, which represents $2 \leq \mathrm{FC}<4$; and false discovery rate (q-value) was set at the level of $10 \%$. The resulting gene list was filtered using $W>10$ (which represents $\mathrm{FC}=2$ in all five time-points) and $\mathrm{q}<10$.

\section{Pathway analysis}

The pathway analysis, which identifies the most relevant biological processes to a specific list of candidate genes, was conducted using the Ingenuity Pathways Knowledge Base tool (IPA, Ingenuity Systems, Inc., Redwood City, CA.) as described previously [19,30]. For this analysis we generated individual gene lists for each time point, therefore the cutoffs for the score value were also set individually and were equal to the number of tested species in a particular time point (Additional file 4). Gene symbols from the resulting gene lists were submitted to the IPA and analyzed using "Tox Lists" tool. Significance of the identified pathways was tested by the IPA imbedded Fisher Exact test p-value [31].

\section{Automated literature search}

PubMatrix (multiplex literature mining tool) analysis [3] was conducted as described previously [32]. We restricted our search to human symbols approved by HUGO Gene Nomenclature Committee (HGNC), which were enriched by all aliases and former (discontinued) symbols for selected candidate genes (http://www.genenames.org). The symbols with the hyphen and double names separated by a slash were filtered out. As a result our 47 candidates were combined with 109 aliases and cross-referenced with terms "kidney injury", "kidney ischemia reperfusion injury", and "kidney transplantation". The biomedical literature reference count for a given gene was represented by the highest number among all aliases of a given gene. The genes with number of references $\mathrm{N} \geq 5$ were considered to have established association with kidney IRI, $5>\mathrm{N}>0$ were considered novel, and $\mathrm{N}=0$ were considered new.

\section{Evaluating the effects of the weighted scoring algorithm}

To evaluate the effect of our new algorithm on the selection of gene candidates, we compared the overall species 
signal contribution to the selected gene candidates with candidates that were filtered out by our method. The fraction of rodent model contribution and the fraction of human model contribution were used as the endpoints for comparison.

Oxygen-Glucose deprivation of human kidney (HK-2) cells Human, kidney proximal tubule (HK-2) cells were purchased from American Type Culture Collection (ATCC ${ }^{\oplus}$, cat\# CRL-2190, LOT: 59538658) and grown on T75 flask until confluent.

The hypoxia experiment was conducted as described previously [33]. Briefly, cells were washed with warmed PBS and OGD solutions, and covered with oxygen deprived OGD solution. The airspace in the flask was flashed with nitrogen to remove remaining oxygen. The flack was capped and placed into HeraCell incubator at $1 \% \mathrm{O}_{2}$ and $37^{\circ} \mathrm{C}$. After $2 \mathrm{~h}$ flasks were removed and OGD solution was either replaced with growing media (24 h recovery) or treated with lysis buffer from mir-Vana kit ( 0 h recovery) for RNA extraction.

\section{Real-time RT-PCR}

Transcript levels of TYROBP in HK-2 cells from 2 independent experiments were measured as previously described with slight modification according to new manufacturer protocols [29]. Briefly, the 384-well microtiter plate setting of a $\mathrm{ViiA}^{\mathrm{Tm}} 7$ Real-Time PCR System (Applied Biosystems) was employed. TaqMan ${ }^{\circledR}$ Predeveloped Assay Reagent human $\beta$-actin (REF 4326315E, probe dye VIC-MGB) was used as an internal control for normalization. TaqMan ${ }^{\oplus}$ Gene Expression assay for human TYROBP was purchased from Applied Biosystems Inc. (Hs00182426 m1). All experimental protocols were based on manufacturer's recommendation using the TaqMan ${ }^{\circledR}$ Universal Master Mix II (P/N 4440039). A relative quantitative method was used to calculate TYROBP transcript levels relative to the actin expression. The significance of the obtained difference in signal between sham and hypoxia treated cells was assessed using t-test where $\mathrm{p}<0.05$ was considered significant.

\section{Additional files}

Additional file 1: The working examples of new scoring algorithm. Additional file 2: List of 75 gene candidates identified by chi-square test. Additional file 3: List of 116 gene candidates identified by new scoring algorithm.

Additional file 4: List of $\mathbf{4 7}$ gene candidates identified by both chi-square test and new scoring algorithm.

Additional file 5: List of interactive networks identified by the Ingenuity software.

Additional file 6: Figure S1. Species correction using new algorithm. Additional file 7: Amplification plots of real time PCR studies.
Competing interests

The authors declare that they have no competing interests.

\section{Authors' contributions}

DNG, HR and SQY conceived of the study. LQZ and DPH participated in its design. DRC carried out mouse and tissue culture studies. PH carried out real time PCR studies. DNG and SQY did statistical analyses of all data and drafted the manuscript. All authors read and approved the final manuscript.

\section{Acknowledgements}

This work was in part supported by mini grant "Molecular mechanisms of ischemia and reperfusion of human renal allograft" from National Kidney Foundation of Maryland to DNG.

\section{Author details}

${ }^{1}$ Division of Experimental and Translational Genetics, Department of Pediatrics, Children's Mercy Hospitals and Clinics, University of Missouri School of Medicine, Kansas City, MO, USA. ${ }^{2}$ Department of Biomedical and Health Informatics, University of Missouri School of Medicine, Kansas City, MO, USA. ${ }^{3}$ Omics Analysis Unit, Core of Genetic Research, Division of Experimental and Translational Genetics, Department of Pediatrics, Children's Mercy Hospitals and Clinics, University of Missouri-Kansas City School of

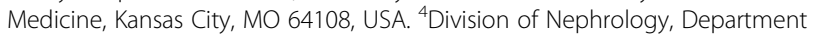
of Medicine, Johns Hopkins University, Baltimore, MD, USA.

Received: 12 June 2013 Accepted: 11 October 2013

Published: 24 October 2013

\section{References}

1. Tilney NL, Guttmann RD: Effects of initial ischemia/reperfusion injury on the transplanted kidney. Transplantation 1997, 64(7):945-947.

2. Terasaki PI, Cecka JM, Gjertson DW, Takemoto S: High survival rates of kidney transplants from spousal and living unrelated donors. N Engl J Med 1995, 333(6):333-336.

3. Becker KG, Hosack DA, Dennis G Jr, Lempicki RA, Bright TJ, Cheadle C, Engel J: PubMatrix: a tool for multiplex literature mining. BMC Bioinforma 2003, 4:61.

4. Facio FN Jr, Sena AA, Araujo LP, Mendes GE, Castro I, Luz MA, Yu L, Oliani SM, Burdmann EA: Annexin 1 mimetic peptide protects against renal ischemia/reperfusion injury in rats. J Mol Med 2011, 89(1):51-63.

5. Wever KE, Wagener FA, Frielink C, Boerman OC, Scheffer GJ, Allison A, Masereeuw $R$, Rongen GA: Diannexin protects against renal ischemia reperfusion injury and targets phosphatidylserines in ischemic tissue. PLoS One 2011, 6(8):e24276.

6. Cheng CW, Rifai A, Ka SM, Shui HA, Lin YF, Lee WH, Chen A: Calciumbinding proteins annexin $\mathrm{A} 2$ and $\mathrm{S} 100 \mathrm{~A} 6$ are sensors of tubular injury and recovery in acute renal failure. Kidney Int 2005, 68(6):2694-2703.

7. Lal-Nag M, Morin PJ: The claudins. Genome Biol 2009, 10(8):235.

8. Hewitt KJ, Agarwal R, Morin PJ: The claudin gene family: expression in normal and neoplastic tissues. BMC Cancer 2006, 6:186.

9. Kieran NE, Doran PP, Connolly SB, Greenan MC, Higgins DF, Leonard M, Godson C, Taylor CT, Henger A, Kretzler M, et al: Modification of the transcriptomic response to renal ischemia/reperfusion injury by lipoxin analog. Kidney Int 2003, 64(2):480-492.

10. Yamanishi Y, Kitaura J, Izawa K, Kaitani A, Komeno Y, Nakamura M, Yamazaki S, Enomoto Y, Oki T, Akiba H, et al: TIM1 is an endogenous ligand for LMIR5/CD300b: LMIR5 deficiency ameliorates mouse kidney ischemia/ reperfusion injury. J Exp Med 2010, 207(7):1501-1511.

11. Krishnamoorthy A, Ajay AK, Hoffmann D, Kim TM, Ramirez V, Campanholle G, Bobadilla NA, Waikar SS, Vaidya VS: Fibrinogen beta-derived Bbeta(1542) peptide protects against kidney ischemia/reperfusion injury. Blood 2011, 118(7):1934-1942.

12. Maeda M, Carpenito C, Russell RC, Dasanjh J, Veinotte LL, Ohta H, Yamamura T, Tan R, Takei F: Murine CD160, Ig-like receptor on NK cells and NKT cells, recognizes classical and nonclassical MHC class I and regulates NK cell activation. J Immunol 2005, 175(7):4426-4432

13. Yim D, Jie HB, Lanier LL, Kim YB: Molecular cloning, gene structure, and expression pattern of pig immunoreceptor DAP12. Immunogenetics 2000, 51(6):436-442. 
14. Kusaka M, Yamada K, Kuroyanagi Y, Terauchi A, Kowa H, Kurahashi H, Hoshinaga K: Gene expression profile in rat renal isografts from brain dead donors. Transplant Proc 2005, 37(1):364-366.

15. Yuen PS, Jo SK, Holly MK, Hu X, Star RA: Ischemic and nephrotoxic acute renal failure are distinguished by their broad transcriptomic responses. Physiol Genomics 2006, 25(3):375-386

16. Hanto DW, Maki T, Yoon MH, Csizmadia E, Chin BY, Gallo D, Konduru B, Kuramitsu K, Smith NR, Berssenbrugge A, et al: Intraoperative administration of inhaled carbon monoxide reduces delayed graft function in kidney allografts in Swine. Am J Transplant 2010, 10(11):2421-2430

17. Vinas JL, Sola A, Jung M, Mastora C, Vinuesa E, Pi F, Hotter G: Inhibitory action of Wnt target gene osteopontin on mitochondrial cytochrome $c$ release determines renal ischemic resistance. Am J Physiol Renal Physiol 2010, 299(1):F234-F242.

18. Naesens M, Li L, Ying L, Sansanwal P, Sigdel TK, Hsieh SC, Kambham N, Lerut E, Salvatierra O, Butte AJ, et al: Expression of complement components differs between kidney allografts from living and deceased donors. J Am Soc Nephrol 2009, 20(8):1839-1851.

19. Grigoryev DN, Liu M, Hassoun HT, Cheadle C, Barnes KC, Rabb H: The local and systemic inflammatory transcriptome after acute kidney injury. J Am Soc Nephrol 2008, 19(3):547-558.

20. Liu M, Grigoryev DN, Crow MT, Haas M, Yamamoto M, Reddy SP, Rabb H: Transcription factor Nrf2 is protective during ischemic and nephrotoxic acute kidney injury in mice. Kidney Int 2009, 76(3):277-285.

21. Grigoryev DN, Ko GJ, Watkins T, Cheadle C, Barnes KC, Rabb H: Molecular signature of reperfusion injury in human renal allografts. J Am Soc Nephrol 2009, 20(Suppl):12A.

22. Cheadle C, Watkins T, Ehrlich E, Barnes K, Gaber AO, Hemmerich S, Rabb H: Effects of anti-adhesive therapy on kidney biomarkers of ischemia reperfusion injury in human deceased donor kidney allografts. Clin Transplant 2011, 25(5):766-775.

23. Ko GJ, Grigoryev DN, Linfert D, Jang HR, Watkins T, Cheadle C, Racusen L, Rabb H: Transcriptional analysis of kidneys during repair from AKI reveals possible roles for NGAL and KIM-1 as biomarkers of AKI-to-CKD transition. Am J Physiol Renal Physiol 2010, 298(6):F1472-F1483.

24. Tusher VG, Tibshirani R, Chu G: Significance analysis of microarrays applied to the ionizing radiation response. Proc Natl Acad Sci U S A 2001, 98(9):5116-5121.

25. Larsson O, Wahlestedt C, Timmons JA: Considerations when using the significance analysis of microarrays (SAM) algorithm. BMC Bioinforma 2005, 6:129.

26. Polotsky WY, Savransky V, Bevans-Fonti S, Reinke C, Li J: Grigoryev DN. Shimoda LA: Intermittent and Sustained Hypoxia Induce a Similar Gene Expression Profile in the Human Aortic Endothelial Cells. Physiol Genomics; 2010.

27. Chen R, Li L, Butte AJ: AlLUN: reannotating gene expression data automatically. Nat Methods 2007, 4(11):879.

28. Kodama K, Horikoshi M, Toda K, Yamada S, Hara K, Irie J, Sirota M, Morgan $A A$, Chen $R$, Ohtsu $H$, et al: Expression-based genome-wide association study links the receptor CD44 in adipose tissue with type 2 diabetes. Proc Natl Acad Sci U S A 2012, 109(18):7049-7054.

29. Grigoryev DN, Ma SF, Shimoda LA, Johns RA, Lee B, Garcia JG: Exon-based mapping of microarray probes: recovering differential gene expression signal in underpowered hypoxia experiment. Mol Cell Probes 2007, 21(2):134-139.

30. Hassoun HT, Lie ML, Grigoryev DN, Liu M, Tuder RM, Rabb H: Kidney ischemia-reperfusion injury induces caspase-dependent pulmonary apoptosis. Am J Physiol Renal Physiol 2009, 297(1):F125-F137.

31. Jimenez-Marin A, Collado-Romero M, Ramirez-Boo M, Arce C, Garrido JJ: Biological pathway analysis by ArrayUnlock and Ingenuity Pathway Analysis. BMC proceedings 2009, 3(Suppl 4):S6.
32. Grigoryev DN, Ma SF, Irizarry RA, Ye SQ, Quackenbush J, Garcia JG: Orthologous gene-expression profiling in multi-species models: search for candidate genes. Genome Biol 2004, 5(5):R34

33. Rizvi M, Jawad N, Li Y, Vizcaychipi MP, Maze M, Ma D: Effect of noble gases on oxygen and glucose deprived injury in human tubular kidney cells. Exp Biol Med (Maywood) 2010, 235(7):886-891.

doi:10.1186/1471-2369-14-231

Cite this article as: Grigoryev et al:: Meta-analysis of molecular response of kidney to ischemia reperfusion injury for the identification of new candidate genes. BMC Nephrology 2013 14:231.

\section{Submit your next manuscript to BioMed Central and take full advantage of:}

- Convenient online submission

- Thorough peer review

- No space constraints or color figure charges

- Immediate publication on acceptance

- Inclusion in PubMed, CAS, Scopus and Google Scholar

- Research which is freely available for redistribution

Submit your manuscript at www.biomedcentral.com/submit
C BioMed Central 